\title{
Study on Prediction Model of Roof Falling based on Crisis Signs in Coal Mine
}

\author{
Zhong-an Jiang, Ya Chen \& Cong Tan \\ University of Science and Technology, Beijing \\ Civil and Environment Engineering School, China
}

\begin{abstract}
To effectively prevent roof falling to cause heavy losses in coal mine, the paper selected crisis signs as prediction indicators before roof disaster. Through counting and analyzing accident cases, twentythree indicators were identified, which can be used for prediction. Given to the characteristic of these crisis indicators, BP neural network was determined as our prediction model and probability model was selected as our data model. The experimental results confirm the effectiveness of our proposed model.
\end{abstract}

KEYWORD: Prediction model: Roof falling: Crisis signs: BP neural network

\section{INTRODUCTION}

Roof falling is one of the factors to cause accident in coal mine. As the ruinous destructiveness of mine roof falling, the accident can have a devastating impact on workers. For example, in the afternoon of August 16, 2012, there was a roof falling accident, in Ruifeng coal mine, Fugu Coutry, Shaanxi Province. By an initial check, among 20 people on duty working underground, only two were safe to up, another 18 workers were trapped underground.

As the complex and dynamic nature of this event, this early prediction of this crisis attracted many researchers attentions. Wang et al. analyzed the symptom of roof falling [Gregory et al. 1999 and Duzgun et al. 2004]. Wang et al. [2009] proposed early monitoring system to transfer the monitor problem into a prediction problem. These works study some of the possible symptoms that may cause roof falling and lack an overall review and summarization on these symptoms. In addition, existing prediction models simply utilize machine learning algorithms, without taking into consideration the properties of the available dataset.

To overcome the limitation of existing works, in this paper, we proposed a model-based roof falling early prediction expert system. Specifically, we first summarize all the roof falling symptoms. Then, we propose the roof falling prediction method. As the number of domain experts is usually very small, to introduce the uncertainty of expert opinions, we propose a data generation method that can generate input data for the artificial neural network model. Finally, a neural network algorithm is applied to predict the roof falling probability.

To the best of our knowledge, this is the first work that complementarily summarizes all possible roof falling crisis signs. In addition, roof falling expert system architecture is designed and the data preparation and prediction model are proposed.

The remainder of this paper is organized as follows. Section 2 discusses the symptoms and crisis indicators. Section 3 presents a formulation of roof falling prediction and provides algorithms resulting from artificial neural network model. Section 4 presents an experimental evaluation and system interfaces of our approach. This paper ends with some discussion and brief conclusions in section 5 .

\section{ROOF FALLING SYMPTOMS}

One of the main goals of this paper is to summarize the possible symptoms, build crisis index systems and provide a quantitative measuring approach. In this section, we first introduce roof falling crisis indicators are a system of symptom index before the occurrence of roof falling. We choose its gradual process as a reference, and then discuss the symptoms we used for early prediction.

Table 1 the statistic of roof falling crisis signs

\begin{tabular}{|c|c|}
\hline Type of signs of roof crisis & Number of accident \\
\hline Sound phenomenon & 45 \\
\hline Dregs phenomenon & 33 \\
\hline Anchor and bolt snapping & 19 \\
\hline Roof cracks & 9 \\
\hline Roof watering & 14 \\
\hline
\end{tabular}




\subsection{Determine the index system of roof falling crisis}

Based on the analysis from 1960 to 2012 between 100 accident cases of roof falling, and a large amount of literature, we statistically analysis according its documented signs before roof falling appeared, shown as Table 1.

It can be seen that the signs of roof falling are the typical characteristics. It appears to play an important role in judging whether or not the occurrence of accident. Therefore, we identified seven main indicators: beep, dregs, spall increased, roof cracks and separation, the amount of roof water leaching, anchor and bolt snapping, the whole roof to sink or pot-like appearance and shoulders fracture.

As the content of crisis indicators is a sensory and the description is not specific, these indicators should be refined to make more operational and guidance in order to facilitate identification. We detailed these indicators, as shown in Fig.2.

\subsection{Quantitative indicators of crisis signs}

Roof falling accident is often caused by many factors. And there will be a variety of general crisis symptoms. We assume that the selected 23 indicators are independent and scores may result the likelihood of a roof falling accident, given the numerical between 0 and 1 , the greater the value, the higher the risk.

\section{PREDICTION MODEL OF ROOF FALLING}

\subsection{BP neural network model}

BP neural network (BPNN) is good for nonprecision calculations and obfuscation, and it is also suitable for large-scale data on sensory indicators and parallel processing. Meanwhile, artificial neural networks have capabilities of strong self-learning, association, fault tolerance, and good adaptability and self-organization.

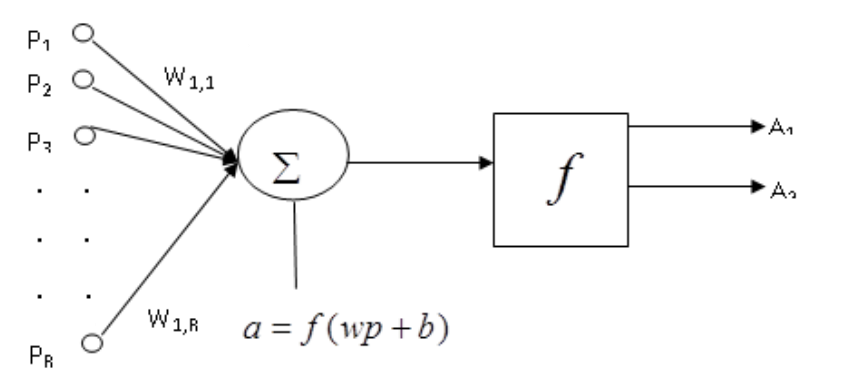

Figure 1 Artificial Neural Network

BPNN is a multilayer feed-forward neural networks, which are passed neuron function S-type. Output is continuous between the value of 0 and 1 . It can be achieved from the input to the output of any non-linear mapping, shown in Figure 2.

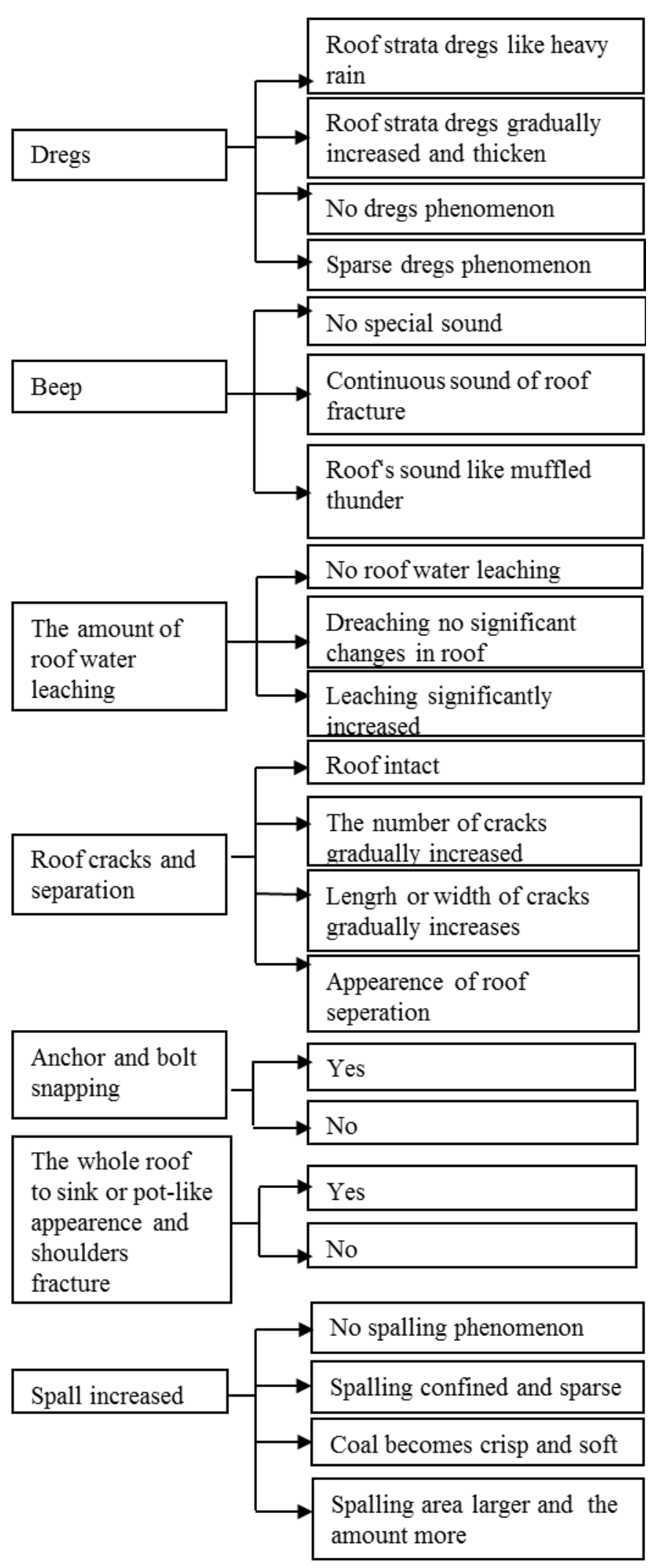

Figure 2 Roof falling symptoms

It has $\mathrm{R}$ inputs. Each input has an appropriate weight $\mathrm{W}$. $\mathrm{R}$ is connected with the lower layer, which represents the transfer function of input/output relationship. $A_{1}$ and $A 2$ are the outputs of the output value of the nodes.

Generally, we should follow the following steps to build an artificial neural network:

1. Determine the number of layers. BP neural network contains an input layer, one or more hidden layer, and an output layer. Normally, 
there are three layers. Then determine the number of nodes in the input layer, hidden layer node and output nodes according to the specific problem.

2. Select the network training function, from the input layer to the hidden layer and from the hidden layer to the output layer.

3 . Enter the study sample and the output target vector, then determine the number of training, training step, training error and accuracy.

4. Through neural network learning, to see whether or not meet the default requirement, if not, you will need to re-adjust the network parameters and functions, until compliance with the convergence precision.

5. Finally get the desired results through learning the network model.

For roof falling crisis, there are 23 input layer nodes. Network setup function is newff(), input layer transfer function is tansig(), the transfer function of the output layer is $\log \operatorname{sig}()$, the number of network training is 1000 times, the performance objective is 0.01 . there are two output nodes, the output values of " 0 " and " 1 ". " 0 " indicates that an accident will not happen and " 1 " indicates that an accident will happen. We choose the weight associated with the output " 1 " as the predictive value.

\subsection{Model and Data Generation}

\subsubsection{Problem definition}

To formally define the problem studied in the article, we denote the roof falling occurrence by $y$ and denote all factors which may impact the occurrence of crises by $x_{I}=\left\{x_{i}: i \in I\right\}$, where $I$ is the index set of factors. Then our problem is translated to determine the probability of the occurrence of the roof falling crisis, $p\left(y \mid x_{I}\right)$, given a set of factor observation $x_{I}$. Based on the Bayesian Rule, $p\left(y \mid x_{i}\right)$ can be transferred to

$$
p\left(y \mid x_{i}\right)=\frac{p\left(x_{i}\right) p\left(x_{i} \mid y\right)}{p(y)}
$$

then the probability of crisis occurs is:

$$
p\left(y \mid x_{I}\right)=\frac{p(y) p\left(x_{I} \mid y\right)}{p\left(x_{I}\right)}
$$

We suppose that the factors are independent with each other, then:

$$
\begin{aligned}
& p\left(x_{I}\right)=\prod_{i \in I} p\left(x_{i}\right) \\
& p\left(x_{I} \mid y_{i}\right)=\prod_{i \in I} p\left(x_{i} \mid y\right)
\end{aligned}
$$

and equation (1) can be translated as:

$$
p\left(y \mid x_{I}\right)=\prod_{i \in I} \frac{p(y) p\left(x_{i} \mid y\right)}{p\left(x_{i}\right)}
$$

\subsection{Data generation}

As the history data for this study is not always available or in a relatively small set. To avoid this shortage of data, we choose domain experts to score the factors which may cause crises. To transfer the expert opinions to $x_{I}$, we generate training data based on the probability distribution specified by experts. Specifically, we choose a number of domain experts to evaluate the probability caused by each factor. The higher is this value, the more possible will the crisis occur. In addition, the value that experts score is in the range of $[0,1]$ with a specified amount of precision ( 2 decimal place) .

In this work, we take normal distribution to generate training data:

$$
f(x)=\frac{1}{\sqrt{2 \pi} \sigma} e^{-\frac{(x-\mu)^{2}}{2 \sigma^{2}}}
$$

where $\mu$ is the score that an expert evaluated the factor and we set $\sigma \leq 0.2$.

This size of the generated vector equals to the number of factors to be evaluated. For each expert's evaluation, a set of binary vectors is generated. The location corresponding to the factor is set as 1 and others are randomly set. We generate the output label (last item in the vector) with the probability of $f(x)$. Note that the last column of the generated vectors represents the occurrence of roof falling.

\section{EXPERIMENTS}

In this section we first describe the experiment setups and the metric used for evaluation. Then, we analyze the experiment results and evaluate the performance of our proposed approaches.

\subsection{Experiment Setups}

There are 23 factors to be evaluated by experts. For each evaluation, we generate 10000 rows of data. For training and testing purpose, we randomly choose $80 \%$ of the data as the training data and take the rest as testing set.

\subsection{Evaluation Metrics}

To evaluate the performance of our algorithm, we make use of Mean Square Error (MSE) to demonstrate the performance of our proposed model. MSE is a statistical accuracy metric which is widely used to measure the prediction quality. 
The definition of MSE is given by the following equation:

$$
\mathrm{MSE}=\frac{1}{n} \sum_{i=1}^{n}\left(\hat{\mathrm{Y}}_{i}-\mathrm{Y}_{i}\right)
$$

where $Y_{i}$ hat and $Y_{i}$ represent the predicted value and the original value respectively.

Precision, recall, F-score [Rijsbergen 1997] are commonly used in evaluating information retrieval and prediction systems. Precision is defined as the ratio of correctly predicted items to the total number of items to be predicted. Recall is defined as the ratio of the number of predicted events to the total number of happened events. F-score is defined as the harmonic mean of above two measures and is calculated by:

$$
F=\frac{2 * \text { Precision } * \text { Recall }}{\text { Precision }+ \text { Recall }}
$$

\subsection{Experimental Results}

To demonstrate the prediction performance of our model, we choose 5 experts to evaluate the symptoms of roof falling and run the experiment 100 rounds. Table 3 lists the minimum and average precision of these experiments. It can be seen that the average precision is above $80 \%$ in terms of precision, recall and F-measure. This result highly confirms the effectiveness of our model.

To demonstrate the predicting precision when choosing different numbers of experts, we compare 4, 8, 16, 32 and 64 experts to run the model. Table 4 shows the MSE values of our model with different number of experts. It has been shown that even with a small number of experts our system can still generate predictions in an acceptable level.

Table 2 The performance of the roof falling prediction system.

\begin{tabular}{|c|c|c|c|}
\hline & Precision & Recall & F-Measure \\
\hline Minimum & $64.39 \%$ & $74.85 \%$ & $70.12 \%$ \\
\hline Average & $81.2 \%$ & $89.34 \%$ & $87.23 \%$ \\
\hline
\end{tabular}

Table 3 The precision of the prediction at different number of experts.

\begin{tabular}{|c|c|c|c|c|c|}
\hline & 4 & 8 & 16 & 32 & 64 \\
\hline MSE & 0.0820 & 0.0357 & 0.0097 & 0.0079 & 0.0002 \\
\hline
\end{tabular}

\section{CONCLUSION AND FUTURE WORK}

In this paper, we summarize all roof falling accident symptoms and propose prediction models for roof falling. One of the main contributions of this work is to incorporate the uncertainty when the number of expert is relatively small and the data generation approach convert expert opinions into the input for BP-ANN. The experimental result confirms the effectiveness of our proposed model.

In fact, there are still many other roof falling symptoms that are not easy to be quantified and we are going to take more of these potential signs considered in the prediction model in the future. In addition, as we discussed in the experiment section, the parameter selecting strategies should also be investigated in our future work.

\section{REFERENCES}

[1] Rijsbergen, C. J. "Information Retrieval," Dept. of Computer Science, University of Glasgow, Second edition. 1997

[2] Wang Xuefeng, Zhu Donghua. Research on technology early warning system based on dynamic information monitoring. Journal of Beijing Institute Technology. 2009.

[3] Christopher Mark, Application of the coal mine roof-rating (CMMR) to extend cuts. Mine Engineering 1999 51(4):5256

[4] Gregory M. Molinda,1 Christopher Mark, and Dennis Dolinar Assessing coal mine roof stability through roof fall analysis, National Institute for Occupational Safety and Health, Technology News 493, 2001 Aug :1-2

[5] Duzgun, H.S.B. and Einstein, H.H. Assessment and management of roof fall risks in underground coal mines, Safety Science, 42(1):23-41, 2004 